OPEN ACCESS

Edited by:

Alberto Sanz,

Newcastle University, United Kingdom

Reviewed by:

lain P. Hargreaves,

Liverpool John Moores University,

United Kingdom

Carlos Santos Ocana

Universidad Pablo de Olavide, Spain

*Correspondence:

Fabien Pierre

fabien.pierre/@univ-grenoble-alpes.fr

Specialty section

This article was submitted to Mitochondrial Research,

a section of the journal

Frontiers in Physiology

Received: 28 March 2017 Accepted: 08 June 2017

Published: 22 June 2017

Citation:

Pierrel F (2017) Impact of Chemical Analogs of 4-Hydroxybenzoic Acid on Coenzyme Q Biosynthesis: From Inhibition to Bypass of Coenzyme Q Deficiency. Front. Physiol. 8:436. doi: 10.3389/fphys.2017.00436

\section{Impact of Chemical Analogs of 4-Hydroxybenzoic Acid on Coenzyme $Q$ Biosynthesis: From Inhibition to Bypass of Coenzyme Q Deficiency}

\author{
Fabien Pierrel * \\ Centre National de la Recherche Scientifique, Grenoble INP, TIMC-IMAG, University Grenoble Alpes, Grenoble, France
}

Coenzyme $Q$ is a lipid that participates to important physiological functions. Coenzyme $Q$ is synthesized in multiple steps from the precursor 4-hydroxybenzoic acid. Mutations in enzymes that participate to coenzyme $Q$ biosynthesis result in primary coenzyme $Q$ deficiency, a type of mitochondrial disease. Coenzyme $Q_{10}$ supplementation of patients is the classical treatment but it shows limited efficacy in some cases. The molecular understanding of the coenzyme $Q$ biosynthetic pathway allowed the design of experiments to bypass deficient biosynthetic steps with analogs of 4-hydroxybenzoic acid. These molecules provide the defective chemical group and can reactivate endogenous coenzyme $Q$ biosynthesis as demonstrated recently in yeast, mammalian cell cultures, and mouse models of primary coenzyme $Q$ deficiency. This mini review presents how the chemical properties of various analogs of 4-hydroxybenzoic acid dictate the effect of the molecules on CoQ biosynthesis and how the reactivation of endogenous coenzyme $Q$ biosynthesis may achieve better results than exogenous $\mathrm{CoQ}_{10}$ supplementation.

Keywords: coenzyme Q, CoQ deficiency, mitochondrial disease, 4-hydroxybenzoic acid, para-aminobenzoic acid, biosynthesis, chemical analogs, bioavailability

\section{INTRODUCTION}

Coenzyme Q (CoQ, compound $\mathbf{1}$ on Figure 1), also known as ubiquinone, is a lipid conserved from proteobacteria to humans. $\mathrm{CoQ}$ is composed of a benzoquinone ring that is attached to a polyisoprenyl tail of various length (six isoprenyl units in Saccharomyces cerevisiae hence $\mathrm{CoQ}_{6}$, ten units in humans, hence $\mathrm{CoQ}_{10}$ ). The benzoquinone ring is redox active and exchanges two electrons and two protons between the oxidized and reduced forms of $\mathrm{CoQ}$, which play numerous roles in cellular physiology (Bentinger et al., 2010; Wang and Hekimi, 2016).

In eukaryotic cells, the biosynthesis of CoQ takes place at the mitochondrial inner membrane (Wang and Hekimi, 2013a) and also possibly in the Golgi apparatus (Mugoni et al., 2013). 4hydroxybenzoic acid $(4-\mathrm{HB}, 2)$ is the precursor of the benzoquinone ring of CoQ. 4- $\mathrm{HB}$ is first prenylated by Coq2 (UbiA in bacteria) and then, a total of seven reactions-one decarboxylation, three hydroxylation, and three methylation-produce the fully substituted benzoquinone ring of $\mathrm{CoQ}$ (Figure 1). Even though the structure of $\mathrm{CoQ}$ was established almost 60 years ago (Lester et al., 1958; Morton, 1958), the identity of the enzymes that catalyze the decarboxylation reaction and one of the three hydroxylation reaction is still elusive in eukaryotes (Kawamukai, 2016). In addition, the pathway that converts tyrosine (3) into $4-\mathrm{HB}$ is poorly characterized and the last 


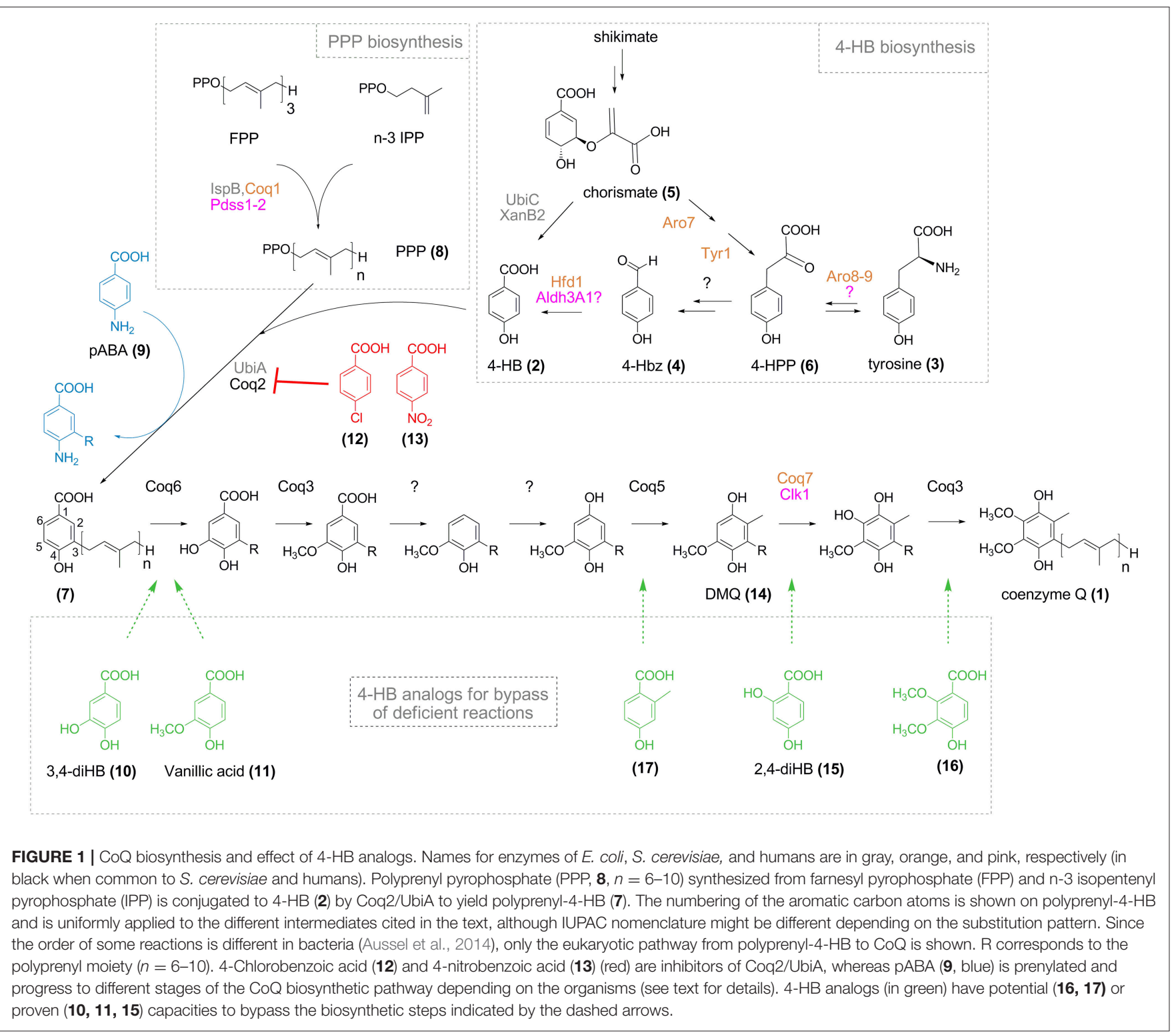

reaction, the oxidation of 4-hydroxybenzaldehyde $(4-\mathrm{Hbz}, 4)$ to 4-HB was only recently elucidated in S. cerevisiae (Payet et al., 2016; Stefely et al., 2016).

Primary $\mathrm{CoQ}_{10}$ deficiency, caused by mutations in genes involved in CoQ biosynthesis, is a rare condition with a heterogeneous clinical spectrum. Mutations in PDSS1, PDSS2, COQ2, COQ4, COQ6, COQ7, COQ9, ADCK3, and ADCK4 have been identified to date (Acosta et al., 2016). CoQ deficiency represents one of the few mitochondrial disorder that is treatable (Hirano et al., 2012), although not all patients respond to oral $\mathrm{CoQ}_{10}$ supplementation (Hirano et al., 2012). The success of the treatment is influenced by the advance of the disease at the time when $\mathrm{CoQ}_{10}$ supplementation is initiated (Acosta et al., 2016). The poor bioavailability of $\mathrm{CoQ}_{10}$ also contributes to the variable results of $\mathrm{CoQ}_{10}$ supplementation. Indeed, the lipophilic nature of $\mathrm{CoQ}_{10}$ is thought to limit its distribution in the human body and its transport to the mitochondrial inner-membrane (Bentinger et al., 2003; Lopez et al., 2010; Hirano et al., 2012).

This mini-review will discuss how various 4-HB analogs impact CoQ biosynthesis and how some of them bypass altered biosynthetic steps, as first demonstrated in $S$. cerevisiae by my group and that of Catherine Clarke (Ozeir et al., 2011; Xie et al., 2012). I will also present the potential benefits and limitations of using analogs of 4 - $\mathrm{HB}$ over $\mathrm{CoQ}_{10}$ supplementation to treat $\mathrm{CoQ}_{10}$ deficiency linked to mutations in specific genes.

\section{BIOSYNTHESIS OF 4-HB, THE NATURAL PRECURSOR OF THE AROMATIC RING OF COQ}

4-HB together with $4-\mathrm{Hbz}$ have been suspected early on as potential precursors of the benzoquinone ring of CoQ in animals, 
yeast, and bacteria (Parson and Rudney, 1964). In Escherichia coli, 4-HB is produced by a chorismate pyruvate-lyase reaction catalyzed by UbiC (Nichols and Green, 1992; Siebert et al., 1992), which substrate is chorismic acid (5), an intermediate of the shikimate pathway that feeds the biosynthesis of aromatic amino acids (Lawrence et al., 1974). However, many proteobacteria that synthesize CoQ lack an $u b i C$ homolog and the widespread $x a n B 2$ gene was recently shown to encode a bifunctional enzyme that converts chorismate into either 4-HB or 3-HB (Zhou et al., 2013). Animals do not possess the shikimate pathway and derive 4-HB from tyrosine and phenylalanine (Olson et al., 1965; Olson, 1966), via a pathway that remains putative (Clarke, 2000), but potentially implicates para-coumarate (Xie et al., 2015). In the plant Arabidopsis thaliana, phenylalanine and tyrosine independently contribute to the synthesis of 4-HB (Block et al., 2014). Only the pathway originating from phenylalanine has been partially characterized and involves $\beta$-oxidation in peroxisomes (Block et al., 2014).

Unlike bacteria, $S$. cerevisiae does not produce $4-\mathrm{HB}$ in a single step from chorismate. Instead, pathways from shikimate or exogenous tyrosine converge at 4-hydroxyphenyl pyruvate (4$\mathrm{HPP}, 6$ ), which is further converted to $4-\mathrm{Hbz}$ via uncharacterized steps (Payet et al., 2016) (Figure 1). As a final reaction, 4- $\mathrm{Hbz}$ is oxidized to $4-\mathrm{HB}$ by the aldehyde dehydrogenase Hfd1 (Payet et al., 2016; Stefely et al., 2016). Since the human homolog $A L D H 3 A 1$ complements the defects of yeast $\Delta h f d 1$ cells for CoQ biosynthesis and respiratory growth (Payet et al., 2016; Stefely et al., 2016), the oxidation of $4-\mathrm{Hbz}$ to $4-\mathrm{HB}$ may also take place in humans, as suspected from early results in animals (Parson and Rudney, 1964). The elucidation of the human pathway from tyrosine to $4-\mathrm{HB}$ is important as mutations in participating genes may result in CoQ deficiency, which may be compensated by supplying 4-HB. Indeed, exogenous $4-\mathrm{HB}$ rescues the levels of CoQ in mutants that disrupt 4-HB biosynthesis in bacteria, yeast, and plants (Zhou et al., 2013; Block et al., 2014; Payet et al., 2016; Stefely et al., 2016).

\section{RULES FOR THE PRENYLATION OF 4-HB ANALOGS}

4-HB enters the CoQ biosynthetic pathway via the prenylation of the position 3 catalyzed by Coq 2 in eukaryotes and UbiA in bacteria that yield 3-polyprenyl-4-hydroxybenzoic acid (7). The polyprenyl pyrophosphate (PPP, 8) is formed by Coq1/Pdss1Pdss2 (Kawamukai, 2016) (Figure 1). Aromatic compounds are substrates of UbiA if their carbon C3 is activated by a carboxylic acid moiety on $\mathrm{C} 1$ and a group on $\mathrm{C} 4$ that is electronand hydrogen bond-donor, like hydroxyl or amine groups (Wessjohann and Sontag, 1996). Electron-withdrawing groups on C4, such as nitro and chloro, led to loss of activity of UbiA (Wessjohann and Sontag, 1996; Brandt et al., 2009). Interestingly, substituents in position 5 and 6 of 4 - $\mathrm{HB}$ are tolerated.

These structural requirements are also applicable with the rat Coq2 enzyme since para-aminobenzoic acid (pABA, 9), 3,4-dihydroxybenzoic acid (3,4-diHB, 10), and 4-hydroxy-3methoxybenzoic acid (vanillic acid, 11) were prenylated in cell free extracts, whereas chlorobenzoic acid (12) inhibited the prenyl transferase reaction (Alam et al., 1975; Nambudiri et al., 1977). Furthermore, 4-nitrobenzoic acid (4-NB, 13) was shown to be a competitive inhibitor of Coq2 and to decrease CoQ biosynthesis in mammalian cell cultures in a dose- and time-dependent manner (Forsman et al., 2010). 4-NB was subsequently used to evaluate how different residual levels of CoQ impact various cellular parameters (Quinzii et al., 2012), contributing to a better understanding of the pathomechanisms underlying primary CoQ deficiency. Altogether, these studies demonstrate that the active site of Coq2-UbiA can accommodate several 4-HB analogs which will act as substrates or inhibitors depending on their chemical properties.

The crystal structures of two UbiA homologs from archaeal thermophiles have recently been reported (Cheng and Li, 2014; Huang et al., 2014). Both membrane-embedded proteins present nine transmembrane helices. In one structure, a lateral portal delineated by two transmembrane domains was proposed to open to the membrane and allow access to the PPP molecule (Cheng and Li, 2014). Two conserved aspartate-rich motifs are located in a central cavity and coordinate two $\mathrm{Mg}^{2+}$ ions involved in binding the PPP analogs used in co-crystallization experiments. Unfortunately, no details are available regarding the coordination of $4-\mathrm{HB}$ in the active site. Indeed, in one enzyme, 4-HB binding could not be detected by isothermal titration calorimetry and tentative 4-HB modeling clashed with the position occupied by the PPP analog (Huang et al., 2014) whereas in the other enzyme, 4-HB was modeled so that its carboxyl group contacts Arg43 (Cheng and Li, 2014). Yet, the mutation of the corresponding arginine residue in E. coli UbiA did not completely abolish activity (Cheng and Li, 2014), raising doubts about the involvement of this residue in the coordination of 4 -HB.

\section{PABA ADVANCES TO DIFFERENT STAGES OF COQ BIOSYNTHESIS DEPENDING ON THE ORGANISMS}

pABA fulfills the requirements for prenylation by the Coq2UbiA prenyltransferases and labeling experiments demonstrated that pABA is converted to CoQ in S. cerevisiae (Marbois et al., 2010; Pierrel et al., 2010). Consequently, the $C 4$ amino group of pABA must be replaced by a $\mathrm{C} 4$ hydroxyl group and we recently reported that the monooxygenase Coq6 catalyzes this reaction in addition to the previously reported C5-hydroxylation (Ozeir et al., 2015). The L382E mutation in Coq6 severely impairs the C4-deamination reaction but globally maintains the C5-hydroxylation (Ozeir et al., 2015). $\Delta$ coq9 cells accumulate C4-aminated intermediates of the CoQ pathway (Xie et al., 2012; He et al., 2015) but Coq9 plays an indirect role in the C4-deamination as its absence impacts the $\mathrm{C}$-terminal region of Coq6, which is important for the C4-deamination but quite dispensable for the C5-hydroxylation (Ozeir et al., 2015).

So far, pABA was reported to be a precursor of CoQ only in S. cerevisiae. E. coli, the plant Arabidopsis, or mammalian cells do not incorporate pABA into CoQ (Block et al., 2014; 
Xie et al., 2015). In E. coli, prenylated pABA is decarboxylated and hydroxylated to yield 2-amino-3-octaprenylphenol, which was suggested to be a "dead-end" product (Xie et al., 2015). In mammalian cell cultures, pABA decreases CoQ levels (GonzalezAragon et al., 2005; Xie et al., 2015) and unidentified prenylated compounds were previously detected (Alam et al., 1975; Forsman et al., 2010). Indeed, results from my group confirmed a strong decrease of cellular $\mathrm{CoQ}_{9}$ in Chinese Hamster Ovary $(\mathrm{CHO})$ cells and NIH/3T3 fibroblasts (derived from Swiss mouse embryo tissue) treated with pABA (Figure 2). In addition, both cell lines accumulate a major redox compound, which was identified as 4imino-6-demethoxycoenzyme $\mathrm{Q}_{9}$ (IDMQ 9 ) by high resolution mass spectrometry. IDMQ 9 can be reduced to 4-amino-6demethoxycoenzyme $\mathrm{Q}_{9}\left(\mathrm{ADMQ}_{9}\right)$ (Figure 2), similarly to IDMQ $_{6}$ that was previously detected in yeast $\Delta$ coq9 cells (Xie et al., 2012). These results suggest that the C4-deamination is not taking place in mammalian cells and that Clk1-Coq7 cannot hydroxylate IDMQ or ADMQ (I-ADMQ). The properties of I-ADMQ are not known but they may have antioxidant capacities and may also participate to mitochondrial respiration as demonstrated for the closely related molecule demethoxycoenzyme Q (DMQ, 14; Wang and Hekimi, 2013b). Therefore, pABA may not be the most appropriate agent to induce $\mathrm{CoQ}_{10}$ deficiency in cell lines and study the consequential physiological impacts (Gonzalez-Aragon et al., 2005; Duberley et al., 2013, 2014). Instead, 4-NB must be considered for such purposes since it does not form any prenylated products (Forsman et al., 2010; Quinzii et al., 2012).

Mammalian cells do not synthesize pABA contrary to microorganisms, which derive pABA from the shikimate pathway (Botet et al., 2007). pABA is frequently found in nutritional supplements but is rapidly and efficiently eliminated from the human body (Sharma et al., 2014), limiting the risk of interference with CoQ biosynthesis. Accordingly, pABA did not perturb CoQ biosynthesis in mice. Indeed the levels of $\mathrm{CoQ}_{9}$ were maintained in various tissues-heart, brain, lung, spleen, kidney, liver, and skeletal muscle-of C57BL/6 mice injected intraperitoneally with pABA (50 mg/kg/day) for 4 weeks and I$\mathrm{ADMQ}_{9}$ were not detected (unpublished results). Thus, pABA seems to interfere with mammalian CoQ biosynthesis only in cell cultures.

\section{USING 4-HB ANALOGS TO BYPASS DEFICIENT STEPS IN COQ BIOSYNTHESIS}

The possibility to bypass a deficient step in CoQ biosynthesis by providing the defective chemical group within a synthetic analog of 4-HB was first demonstrated with 3,4-diHB and vanillic acid (Ozeir et al., 2011). Both compounds restored CoQ biosynthesis in $S$. cerevisiae cells impaired in C5-hydroxylation because of mutations in coq6 (Ozeir et al., 2011; Doimo et al., 2014). When working with knock-out $S$. cerevisiae mutants, the effect of 4-HB analogs is dependent upon overexpression of Coq8 to stabilize several Coq proteins that are instable in $\Delta$ coq strains (Padilla et al., 2009; Xie et al., 2012; He et al., 2014). 2,4-Dihydroxybenzoic acid (2,4-diHB, 15) bypassed a C6-hydroxylation defect and allowed CoQ6 biosynthesis in $\Delta$ coq7 yeast cells overexpressing Coq8 (Xie et al., 2012). 2,4-diHB increased $\mathrm{CoQ}_{10}$ levels in fibroblasts with an homozygous V141E mutation in COQ7 (Freyer et al., 2015) but was inefficient with the L111P mutation (Wang et al., 2017).

Besides cell cultures, 2,4-diHB was also efficient in mice with an inducible deletion of Coq7 (also called Mclk1). Addition

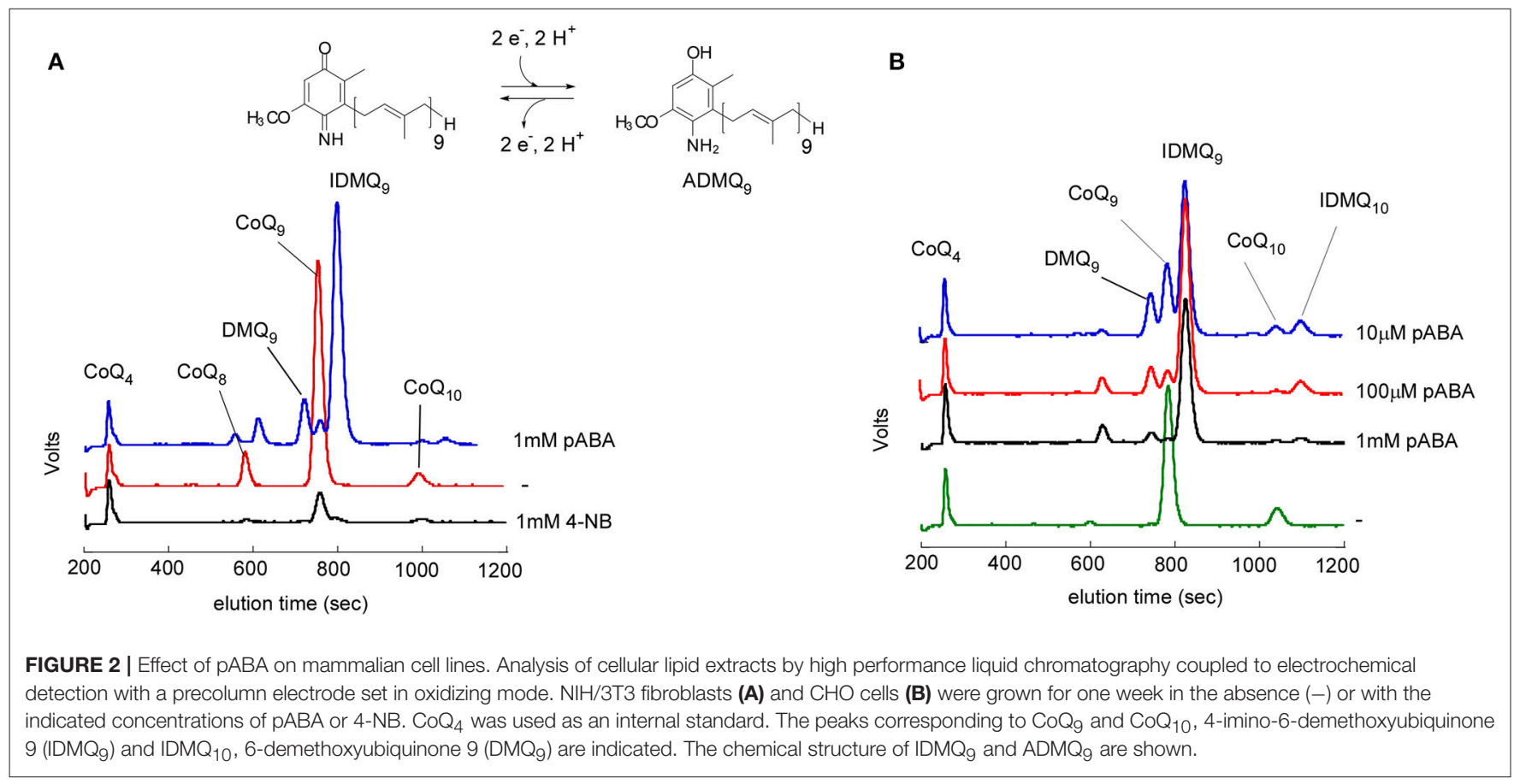


of 2,4-diHB to the drinking water shortly after induction of the Mclk1 deletion increased CoQ 9 levels in heart, kidney, and skeletal muscle and markedly rescued the mutant phenotypes, including mitochondrial respiration, blood lactate levels, and lifespan (Wang et al., 2015). In this model, dietary CoQ 10 supplementation was almost without an effect, likely because of poor tissue uptake except by the liver (Wang et al., 2015). Interestingly, 2,4-diHB was also efficient when provided as a late treatment, i.e., when the symptoms had already developed. Oral 2,4-diHB also proved efficient in the Coq $9^{\mathrm{R} 239 \mathrm{X}}$ mouse, as it increased the kidney $\mathrm{CoQ}_{9}$ content (Luna-Sanchez et al., 2015). $\mathrm{Coq} 9^{\mathrm{R} 239 \mathrm{X}}$ mice have decreased $\mathrm{CoQ}_{9}$ and accumulate $\mathrm{DMQ}_{9}$ (Garcia-Corzo et al., 2013) as the Coq7 reaction is impeded since Coq9 interacts with Coq7 and is thought to facilitate its function (Lohman et al., 2014). Together, these results suggest that 2,4diHB may be beneficial to patients with primary CoQ deficiency due to COQ7 or COQ9 mutations.

Such bypass strategies may be applicable to other cases of primary coenzyme $\mathrm{Q}$ deficiency. COQ6 patients (Heeringa et al., 2011) may respond to vanillic acid or 3,4-diHB (Doimo et al., 2014). Although disease-causing mutations in COQ3 and COQ5 genes have not been reported yet, 2,3-dimethoxy-4hydroxybenzoic acid (16) and 2-methyl-4-hydroxybenzoic acid (17) may, in theory, bypass the requirement for Coq3 and Coq5, respectively. The possibility for the former compound to be prenylated by Coq2 is supported by the in vitro prenylation of the related 2,3,4-trihydroxybenzoic acid (2,3,4-tri-HB) by UbiA (Wessjohann and Sontag, 1996). Altogether, several reactions of the CoQ biosynthetic pathway are potentially amenable to bypass. However, it is impossible to circumvent the $\mathrm{C} 1$ decarboxylation or $\mathrm{C} 1$ hydroxylation reactions with analogs bearing a hydroxyl group on $\mathrm{C} 1$, since the presence of a carboxyl group at $\mathrm{C} 1$ of the benzene ring is indispensable for prenylation (Alam et al., 1975; Wessjohann and Sontag, 1996).

\section{ADVANTAGES OF USING ANALOGS OF 4-HB THAT RESTORE ENDOGENOUS COQ BIOSYNTHESIS OVER EXOGENOUS $\mathrm{COQ}_{10}$ SUPPLEMENTATION}

To replenish CoQ levels in CoQ deficient cells and organisms, the use of bypass $4-\mathrm{HB}$ analogs may be advantageous over $\mathrm{CoQ}_{10}$ supplementation for the following reasons. (i) 4-HB analogs will allow to preserve the endogenous ratio between the major and minor isoforms of CoQ. Indeed, many species have a prominent CoQ isoform ( $\mathrm{CoQ}_{9}$ in rodents, $\mathrm{CoQ}_{10}$ in humans) but also synthesize minor isoforms ( $\mathrm{CoQ}_{10}$ in rodents, $\mathrm{CoQ}_{9}$ in humans). The ratio of both isoforms varies significantly depending on the organs (Turunen et al., 2004), yet, it remains unknown whether these varying ratios have any physiological consequences. (ii) Thanks to their hydrophilic nature, 4-HB analogs may have a superior bioavailability than exogenously supplied $\mathrm{CoQ}_{10}$, which accumulates efficiently in the liver but not in other organs (Miles, 2007). Indeed, $\mathrm{CoQ}_{10}$ supplementation of CoQ deficient mouse models did not increase $\mathrm{CoQ}_{10}$ content in kidney (Saiki et al., 2008) or heart or muscle (Wang et al., 2015). However, a new formulation of $\mathrm{CoQ}_{10}$ demonstrated improved bioavailability as it increased $\mathrm{CoQ}_{10}$ levels in all tested organs of $\mathrm{Coq} 9^{\mathrm{R} 239 \mathrm{X}}$ mice, although to a limited extent for most of them (Garcia-Corzo et al., 2014). (iii) 4-HB analogs may achieve higher CoQ levels in organs than $\mathrm{CoQ}_{10}$ supplementation. 2,4-diHB doubled the kidney $\mathrm{CoQ}_{9}$ content of Coq9 ${ }^{\mathrm{R} 239 \mathrm{X}}$ mice (Luna-Sanchez et al., 2015) and tripled that of Mclk1-deficient mice (Wang et al., 2015), although WT levels were not reached in either model. For comparison, $\mathrm{CoQ}_{10}$ supplementation yielded a $\sim 50 \%$ increase of total kidney $\mathrm{CoQ}\left(\mathrm{CoQ}_{9}+\mathrm{CoQ}_{10}\right)$ in the former model (Garcia-Corzo et al., 2014) and none in the latter (Wang et al., 2015). (iv) CoQ produced from 4-HB analogs should distribute normally between subcellular compartments whereas exogenously supplied $\mathrm{CoQ}_{10}$ has difficulties to reach mitochondria and their inner membrane (Bentinger et al., 2003). (v) Short chains analogs of CoQ like idebenone and decylubiquinone have been reported to increase superoxide production (Genova et al., 2003), but 4-HB analogs are not expected to have such effect since they don't have a redox-active benzoquinone moiety.

\section{POTENTIAL LIMITATIONS TO THE USE OF 4-HB ANALOGS}

The successful restoration of endogenous CoQ biosynthesis by 4-HB analogs depends on several factors. (i) The 4-HB analogs must outcompete endogenous 4 - $\mathrm{HB}$ in the prenylation reaction catalyzed by Coq2. Thus, the $\mathrm{Km}$ of Coq2 for the analog should be in the same range as that for $4-\mathrm{HB}$ or the analog should be substantially more abundant than 4-HB. (ii) Except for the defective CoQ biosynthetic step, all other enzymatic reactions must be maintained. However, many CoQ biosynthetic proteins form a complex in human cells (Floyd et al., 2016) and the abundance of several Coq proteins decreased in multiple tissues of the Coq9 $9^{\mathrm{R} 239 \mathrm{X}}$ mice (Lohman et al., 2014; LunaSanchez et al., 2015), reflecting the instability of an incompletely assembled complex, as already observed in yeast (Hsieh et al., 2007; Xie et al., 2012). (iii) The Coq enzymes must be able to modify unnatural substrates with extra chemical groups. For example, Coq6, which usually hydroxylates 3-polyprenyl-4hydroxybenzoic acid (7), has to hydroxylate 3-polyprenyl-2,4dihydroxybenzoic acid in 2,4-diHB treated cells. (iv) The 4-HB analogs should be retained in the body unlike pABA and must be non-toxic, like vanillic acid which is licensed as a food additive (Gitzinger et al., 2012). However, other analogs may not be as innocuous, since control mice treated with 2,4-diHB gained less body weight than untreated mice (Wang et al., 2015) and high doses of 2,3,4-tri-HB were toxic in cell lines (Herebian et al., 2017).

\section{CONCLUSION}

As demonstrated in yeast, mice, and human cell cultures, 4$\mathrm{HB}$ analogs can bypass deficiencies in some steps of CoQ biosynthesis. 4-HB itself could be used to compensate for defects in the tyrosine to 4-HB pathway. These strategies are only possible thanks to a detailed molecular and genetic 
understanding of the CoQ biosynthetic pathway and efforts must continue to elucidate the steps that remain uncharacterized to date. In specific cases of primary CoQ deficiency, providing 4$\mathrm{HB}$ analogs to reactivate the endogenous production of CoQ may represent a therapeutic alternative to $\mathrm{CoQ}_{10}$ supplementation. Further, investigations with animal models will establish whether this approach is realistic.

\section{AUTHOR CONTRIBUTIONS}

The author confirms being the sole contributor of this work and approved it for publication.

\section{REFERENCES}

Acosta, M. J., Fonseca, L. V., Desbats, M. A., Cerqua, C., Zordan, R., Trevisson, E., et al. (2016). Coenzyme Q biosynthesis in health and disease. Biochim. Biophys. Acta 1857, 1079-1085. doi: 10.1016/j.bbabio.2016.03.036

Alam, S. S., Nambudiri, A. M., and Rudney, H. (1975). 4-Hydroxybenzoate: polyprenyl transferase and the prenylation of 4-aminobenzoate in mammalian tissues. Arch. Biochem. Biophys. 171, 183-190. doi: 10.1016/0003-9861(75)90022-3

Aussel, L., Pierrel, F., Loiseau, L., Lombard, M., Fontecave, M., and Barras, F. (2014). Biosynthesis and physiology of coenzyme Q in bacteria. Biochim. Biophys. Acta 1837, 1004-1011. doi: 10.1016/j.bbabio.2014.01.015

Bentinger, M., Dallner, G., Chojnacki, T., and Swiezewska, E. (2003). Distribution and breakdown of labeled coenzyme $\mathrm{Q}(10)$ in rat. Free Radic. Biol. Med. 34, 563-575. doi: 10.1016/S0891-5849(02)01357-6

Bentinger, M., Tekle, M., and Dallner, G. (2010). Coenzyme Q-biosynthesis and functions. Biochem. Biophys. Res. Commun. 396, 74-79. doi: 10.1016/j.bbrc.2010.02.147

Block, A., Widhalm, J. R., Fatihi, A., Cahoon, R. E., Wamboldt, Y., Elowsky, C., et al. (2014). The origin and biosynthesis of the benzenoid moiety of ubiquinone (Coenzyme Q) in Arabidopsis. Plant Cell 26, 1938-1948. doi: $10.1105 /$ tpc.114.125807

Botet, J., Mateos, L., Revuelta, J. L., and Santos, M. A. (2007). A chemogenomic screening of sulfanilamide-hypersensitive Saccharomyces cerevisiae mutants uncovers $\mathrm{ABZ2}$, the gene encoding a fungal aminodeoxychorismate lyase. Eukaryot. Cell 6, 2102-2111. doi: 10.1128/EC.00266-07

Brandt, W., Brauer, L., Gunnewich, N., Kufka, J., Rausch, F., Schulze, D., et al. (2009). Molecular and structural basis of metabolic diversity mediated by prenyldiphosphate converting enzymes. Phytochemistry 70, 1758-1775. doi: 10.1016/j.phytochem.2009.09.001

Cheng, W., and Li, W. (2014). Structural insights into ubiquinone biosynthesis in membranes. Science 343, 878-881. doi: 10.1126/science.1246774

Clarke, C. F. (2000). New advances in coenzyme Q biosynthesis. Protoplasma 213, 134-147. doi: 10.1007/BF01282151

Doimo, M., Trevisson, E., Airik, R., Bergdoll, M., Santos-Ocana, C., Hildebrandt, F., et al. (2014). Effect of vanillic acid on COQ6 mutants identified in patients with coenzyme Q deficiency. Biochim. Biophys. Acta 1842, 1-6. doi: 10.1016/j.bbadis.2013.10.007

Duberley, K. E., Abramov, A. Y., Chalasani, A., Heales, S. J., Rahman, S., and Hargreaves, I. P. (2013). Human neuronal coenzyme Q10 deficiency results in global loss of mitochondrial respiratory chain activity, increased mitochondrial oxidative stress and reversal of ATP synthase activity: implications for pathogenesis and treatment. J. Inherit. Metab. Dis. 36, 63-73. doi: 10.1007/s10545-012-9511-0

Duberley, K. E., Heales, S. J., Abramov, A. Y., Chalasani, A., Land, J. M., Rahman, S., et al. (2014). Effect of Coenzyme Q supplementation on mitochondrial electron transport chain activity and mitochondrial oxidative stress in Coenzyme Q deficient human neuronal cells. Int. J. Biochem. Cell Biol. 50, 60-63. doi: 10.1016/j.biocel.2014.02.003

\section{FUNDING}

This work was supported by Fondation pour la Recherche Médicale (grant number "DPM20121125553") and by Agence Nationale de la Recherche (grant pABACoQ “ANR-11-JSV8$002 ")$.

\section{ACKNOWLEDGMENTS}

I would like to thank the numerous colleagues with whom I had fruitful interactions over the years. I apologize to the authors whose work could not be discussed due to space limitation.

Floyd, B. J., Wilkerson, E. M., Veling, M. T., Minogue, C. E., Xia, C., Beebe, E. T., et al. (2016). Mitochondrial protein interaction mapping identifies regulators of respiratory chain function. Mol. Cell. 63, 621-32. doi: 10.1016/j.molcel.2016.06.033

Forsman, U., Sjoberg, M., Turunen, M., and Sindelar, P. J. (2010). 4-Nitrobenzoate inhibits coenzyme Q biosynthesis in mammalian cell cultures. Nat. Chem. Biol. 6, 515-517. doi: 10.1038/nchembio.372

Freyer, C., Stranneheim, H., Naess, K., Mourier, A., Felser, A., Maffezzini, C., et al. (2015). Rescue of primary ubiquinone deficiency due to a novel COQ7 defect using 2,4-dihydroxybensoic acid. J. Med. Genet. 52, 779-783. doi: 10.1136/jmedgenet-2015-102986

Garcia-Corzo, L., Luna-Sanchez, M., Doerrier, C., Garcia, J. A., Guaras, A., Acin-Perez, R., et al. (2013). Dysfunctional Coq9 protein causes predominant encephalomyopathy associated with CoQ deficiency. Hum. Mol. Genet. 22, 1233-1248. doi: 10.1093/hmg/dds530

Garcia-Corzo, L., Luna-Sanchez, M., Doerrier, C., Ortiz, F., Escames, G., Acuna-Castroviejo, D., et al. (2014). Ubiquinol-10 ameliorates mitochondrial encephalopathy associated with CoQ deficiency. Biochim. Biophys. Acta 1842, 893-901. doi: 10.1016/j.bbadis.2014.02.008

Genova, M. W., Pich, M. M., Biondi, A., Bernacchia, A., Falasca, A., Bovina, C., et al. (2003). Mitochondrial production of oxygen radical species and the role of coenzyme Q as an antioxidant. Exp. Biol. Med. 228, 506-513. doi: 10.1177/15353702-0322805-14

Gitzinger, M., Kemmer, C., Fluri, D. A., El-Baba, M. D., Weber, W., and Fussenegger, M. (2012). The food additive vanillic acid controls transgene expression in mammalian cells and mice. Nucleic Acids Res. 40:e37. doi: 10.1093/nar/gkr1251

Gonzalez-Aragon, D., Buron, M. I., Lopez-Lluch, G., Herman, M. D., GomezDiaz, C., Navas, P., et al. (2005). Coenzyme, Q., and the regulation of intracellular steady-state levels of superoxide in HL-60 cells. Biofactors 25, 31-41. doi: 10.1002/biof.5520250105

He, C. H., Black, D. S., Nguyen, T. P., Wang, C., Srinivasan, C., and Clarke, C. F. (2015). Yeast Coq9 controls deamination of coenzyme Q intermediates that derive from para-aminobenzoic acid. Biochim. Biophys. Acta 1851, 1227-1239. doi: 10.1016/j.bbalip.2015.05.003

He, C. H., Xie, L. X., Allan, C. M., Tran, U. C., and Clarke, C. F. (2014). Coenzyme Q supplementation or over-expression of the yeast Coq8 putative kinase stabilizes multi-subunit Coq polypeptide complexes in yeast coq null mutants. Biochim. Biophys. Acta 1841, 630-644. doi: 10.1016/j.bbalip.2013.12.017

Heeringa, S. F., Chernin, G., Chaki, M., Zhou, W., Sloan, A. J., Ji, Z., et al. (2011). COQ6 mutations in human patients produce nephrotic syndrome with sensorineural deafness. J. Clin. Invest. 121, 2013-2024. doi: 10.1172/JCI45693

Herebian, D., Seibt, A., Smits, S. H. J., Bunning, G., Freyer, C., Prokisch, H., et al. (2017). Detection of 6-demethoxyubiquinone in CoQ10 deficiency disorders: insights into enzyme interactions and identification of potential therapeutics. Mol. Genet. Metab. S1096-7192(17)30100-2. doi: 10.1016/j.ymgme.2017.05.012

Hirano, M., Garone, C., and Quinzii, C. M. (2012). CoQ(10) deficiencies and MNGIE: two treatable mitochondrial disorders. Biochim. Biophys. Acta 1820, 625-631. doi: 10.1016/j.bbagen.2012.01.006 
Hsieh, E. J., Gin, P., Gulmezian, M., Tran, U. C., Saiki, R., Marbois, B. N., et al. (2007). Saccharomyces cerevisiae Coq9 polypeptide is a subunit of the mitochondrial coenzyme Q biosynthetic complex. Arch. Biochem. Biophys. 463, 19-26. doi: 10.1016/j.abb.2007.02.016

Huang, H., Levin, E. J., Liu, S., Bai, Y., Lockless, S. W., and Zhou, M. (2014). Structure of a membrane-embedded prenyltransferase homologous to UBIAD1. PLoS Biol. 12:e1001911. doi: 10.1371/journal.pbio.1001911

Kawamukai, M. (2016). Biosynthesis of coenzyme Q in eukaryotes. Biosci. Biotechnol. Biochem. 80, 23-33. doi: 10.1080/09168451.2015.1065172

Lawrence, J., Cox, G. B., and Gibson, F. (1974). Biosynthesis of ubiquinone in Escherichia-coli-k-12 - biochemical and genetic characterization of a mutant unable to convert chorismate into 4-hydroxybenzoate. J. Bacteriol. 118, 41-45.

Lester, R. L., Crane, F. L., and Hatefi, Y. (1958). Coenzyme Q - a new group of quinones. J. Am. Chem. Soc. 80, 4751-4752. doi: 10.1021/ja01550a095

Lohman, D. C., Forouhar, F., Beebe, E. T., Stefely, M. S., Minogue, C. E., Ulbrich, A., et al. (2014). Mitochondrial COQ9 is a lipid-binding protein that associates with COQ7 to enable coenzyme Q biosynthesis. Proc. Natl. Acad. Sci. U.S.A. 111, E4697-E4705. doi: 10.1073/pnas.1413128111

Lopez, L. C., Quinzii, C. M., Area, E., Naini, A., Rahman, S., Schuelke, M., et al. (2010). Treatment of $\operatorname{CoQ}(10)$ deficient fibroblasts with ubiquinone, CoQ analogs, and vitamin C: time- and compound-dependent effects. PLoS ONE 5:e11897. doi: 10.1371/journal.pone.0011897

Luna-Sanchez, M., Diaz-Casado, E., Barca, E., Tejada, M. A., Montilla-Garcia, A., Cobos, E. J., et al. (2015). The clinical heterogeneity of coenzyme Q(10) deficiency results from genotypic differences in the Coq9 gene. EMBO Mol. Med. 7, 670-687. doi: 10.15252/emmm.201404632

Marbois, B., Xie, L. X., Choi, S., Hirano, K., Hyman, K., and Clarke, C. F. (2010). para-aminobenzoic acid is a precursor in coenzyme Q(6) biosynthesis in Saccharomyces cerevisiae. J. Biol. Chem. 285, 27827-27838. doi: 10.1074/jbc.M110.151894

Miles, M. V. (2007). The uptake and distribution of coenzyme Q(10). Mitochondrion 7, S72-S77. doi: 10.1016/j.mito.2007.02.012

Morton, R. A. (1958). Ubiquinone. Nature 182, 1764-1767. doi: $10.1038 / 1821764 \mathrm{a} 0$

Mugoni, V., Postel, R., Catanzaro, V., De Luca, E., Turco, E., Digilio, G., et al. (2013). Ubiad 1 is an antioxidant enzyme that regulates eNOS activity by CoQ10 synthesis. Cell 152, 504-518. doi: 10.1016/j.cell.2013.01.013

Nambudiri, A. M. D., Brockman, D., Alam, S. S., and Rudney, H. (1977). Alternate routes for ubiquinone biosynthesis in rats. Biochem. Biophys. Res. Commun. 76, 282-288. doi: 10.1016/0006-291X(77)90723-9

Nichols, B. P., and Green, J. M. (1992). Cloning and sequencing of Escherichia coli ubiC and purification of chorismate lyase. J. Bacteriol. 174, 5309-5316. doi: $10.1128 /$ jb.174.16.5309-5316.1992

Olson, R. E., Dialamieh, G. H., Bentley, R., Springer, C. M., and Ramsey, V. G. (1965). Studies on Coenzyme Q. Pattern of labeling in Coenzyme Q9 after administration of isotopic acetate and aromatic amino acids to rats. J. Biol. Chem. 240, 514-523.

Olson, R. E. (1966). Biosynthesis of ubiquinones in animals. Vitam. Horm. 24, 551-574.

Ozeir, M., Muhlenhoff, U., Webert, H., Lill, R., Fontecave, M., and Pierrel, F. (2011). Coenzyme Q biosynthesis: Coq6 Is required for the C5-hydroxylation reaction and substrate analogs rescue Coq6 deficiency. Chem. Biol. 18, 1134-1142. doi: 10.1016/j.chembiol.2011.07.008

Ozeir, M., Pelosi, L., Ismail, A., Mellot-Draznieks, C., Fontecave, M., and Pierrel, F. (2015). Coq6 Is responsible for the C4-deamination reaction in coenzyme Q biosynthesis in Saccharomyces cerevisiae. J. Biol. Chem. 290, 24140-24151. doi: 10.1074/jbc.M115.675744

Padilla, S., Tran, U. C., Jimenez-Hidalgo, M., Lopez-Martin, J. M., MartinMontalvo, A., Clarke, C. F., et al. (2009). Hydroxylation of demethoxy-Q6 constitutes a control point in yeast coenzyme Q6 biosynthesis. Cell. Mol. Life Sci. 66, 173-186. doi: 10.1007/s00018-008-8547-7

Parson, W. W., and Rudney, H. (1964). Biosynthesis of benzoquinone ring of ubiquinone from p-hydroxybenzaldehyde + p-hydroxybenzoic acid in rat kidney azotobacter vinelandii + bakers yeast. Proc. Natl. Acad. Sci. U.S.A. 51, 444-450. doi: 10.1073/pnas.51.3.444

Payet, L. A., Leroux, M., Willison, J. C., Kihara, A., Pelosi, L., and Pierrel, F. (2016). Mechanistic details of early steps in coenzyme Q biosynthesis pathway in yeast. Cell Chem. Biol. 23, 1241-1250. doi: 10.1016/j.chembiol.2016.08.008
Pierrel, F., Hamelin, O., Douki, T., Kieffer-Jaquinod, S., Muhlenhoff, U., Ozeir, M., et al. (2010). Involvement of mitochondrial ferredoxin and para-aminobenzoic acid in yeast coenzyme Q biosynthesis. Chem. Biol. 17, 449-459. doi: 10.1016/j.chembiol.2010.03.014

Quinzii, C. M., Tadesse, S., Naini, A., and Hirano, M. (2012). Effects of inhibiting CoQ(10) Biosynthesis with 4-nitrobenzoate in human fibroblasts. PLoS ONE 7:e30606. doi: 10.1371/journal.pone.0030606

Saiki, R., Lunceford, A. L., Shi, Y., Marbois, B., King, R., Pachuski, J., et al. (2008). Coenzyme Q10 supplementation rescues renal disease in $\mathrm{Pdss} 2 \mathrm{kd} / \mathrm{kd}$ mice with mutations in prenyl diphosphate synthase subunit 2 . Am. J. Physiol. Renal Physiol. 295, F1535-F1544. doi: 10.1152/ajprenal.904 45.2008

Sharma, R. S., Joy, R. C., Boushey, C. J., Ferruzzi, M. G., Leonov, A. P., and McCrory, M. A. (2014). Effects of Para-Aminobenzoic Acid (PABA) form and administration mode on PABA recovery in 24-hour urine collections. J. Acad. Nutr. Diet. 114, 457-463. doi: 10.1016/j.jand.2013.07.045

Siebert, M., Bechthold, A., Melzer, M., May, U., Berger, U., Schroder, G., et al. (1992). Ubiquinone biosynthesis. Cloning of the genes coding for chorismate pyruvate-lyase and 4-hydroxybenzoate octaprenyl transferase from Escherichia coli. FEBS Lett 307, 347-350. doi: 10.1016/0014-5793(92)80710-X

Stefely, J. A., Kwiecien, N. W., Freiberger, E. C., Richards, A. L., Jochem, A., Rush, M. J., et al. (2016). Mitochondrial protein functions elucidated by multi-omic mass spectrometry profiling. Nat. Biotechnol. 34, 1191-1197. doi: $10.1038 /$ nbt.3683

Turunen, M., Olsson, J., and Dallner, G. (2004). Metabolism and function of coenzyme Q. Biochim. Biophys. Acta 1660, 171-199. doi: 10.1016/j.bbamem.2003.11.012

Wang, Y., and Hekimi, S. (2013a). Molecular genetics of ubiquinone biosynthesis in animals. Crit. Rev. Biochem. Mol. Biol. 48, 69-88. doi: 10.3109/10409238.2012.741564

Wang, Y., and Hekimi, S. (2013b). Mitochondrial respiration without ubiquinone biosynthesis. Hum. Mol. Genet. 22, 4768-4783. doi: 10.1093/hmg/ddt330

Wang, Y., and Hekimi, S. (2016). Understanding ubiquinone. Trends Cell Biol. 26, 367-378. doi: 10.1016/j.tcb.2015.12.007

Wang, Y., Oxer, D., and Hekimi, S. (2015). Mitochondrial function and lifespan of mice with controlled ubiquinone biosynthesis. Nat. Commun. 6, 6393. doi: $10.1038 /$ ncomms7393

Wang, Y., Smith, C., Parboosingh, J. S., Khan, A., Innes, M., and Hekimi, S. (2017) Pathogenicity of two COQ7 mutations and responses to 2,4-dihydroxybenzoate bypass treatment. J. Cell. Mol. Med. doi: 10.1111/jcmm.13154. [Epub ahead of print].

Wessjohann, L., and Sontag, B. (1996). Prenylation of benzoic acid derivatives catalyzed by a transferase from Escherichia coli overproduction: method development and substrate specificity. Angew. Chem. 35, 1697-1699. doi: 10.1002/anie.199616971

Xie, L. X., Ozeir, M., Tang, J. Y., Chen, J. Y., Kieffer-Jaquinod, S., Fontecave, M., et al. (2012). Over-expression of the Coq8 kinase in Saccharomyces cerevisiae coq null mutants allows for accumulation of diagnostic intermediates of the coenzyme Q6 biosynthetic pathway. J. Biol. Chem. 287, 23571-23581. doi: 10.1074/jbc.M112.360354

Xie, L. X., Williams, K. J., He, C. H., Weng, E., Khong, S., Rose, T. E., et al. (2015). Resveratrol and para-coumarate serve as ring precursors for coenzyme Q biosynthesis. J. Lipid Res. 56, 909-919. doi: 10.1194/jlr.M057919

Zhou, L., Wang, J. Y., Wang, J. H., Poplawsky, A., Lin, S. J., Zhu, B. S., et al. (2013). The diffusible factor synthase XanB2 is a bifunctional chorismatase that links the shikimate pathway to ubiquinone and xanthomonadins biosynthetic pathways. Mol. Microbiol. 87, 80-93. doi: 10.1111/mmi.12084

Conflict of Interest Statement: The author declares that the research was conducted in the absence of any commercial or financial relationships that could be construed as a potential conflict of interest.

Copyright (C) 2017 Pierrel. This is an open-access article distributed under the terms of the Creative Commons Attribution License (CC BY). The use, distribution or reproduction in other forums is permitted, provided the original author (s) or licensor are credited and that the original publication in this journal is cited, in accordance with accepted academic practice. No use, distribution or reproduction is permitted which does not comply with these terms. 